\title{
Reduced-Dimension Noncircular-Capon Algorithm for DOA Estimation of Noncircular Signals
}

\author{
Weihua Lv, ${ }^{1,2}$ Huapu Sun, ${ }^{1}$ Xiaofei Zhang, ${ }^{1,3}$ and Dazhuan $X u^{1}$ \\ ${ }^{1}$ College of Electronic and Information Engineering, Nanjing University of Aeronautics and Astronautics, Nanjing 210016, China \\ ${ }^{2}$ Luoyang Optoelectro Technology Development Center, Luoyang 471000, China \\ ${ }^{3}$ National Mobile Communications Research Laboratory, Southeast University, Nanjing 210096, China
}

Correspondence should be addressed to Xiaofei Zhang; fei_zxf@163.com

Received 2 September 2015; Revised 18 November 2015; Accepted 22 November 2015

Academic Editor: Wei Liu

Copyright (C) 2015 Weihua Lv et al. This is an open access article distributed under the Creative Commons Attribution License, which permits unrestricted use, distribution, and reproduction in any medium, provided the original work is properly cited.

\begin{abstract}
The problem of the direction of arrival (DOA) estimation for the noncircular (NC) signals, which have been widely used in communications, is investigated. A reduced-dimension NC-Capon algorithm is proposed hereby for the DOA estimation of noncircular signals. The proposed algorithm, which only requires one-dimensional search, can avoid the high computational cost within the two-dimensional NC-Capon algorithm. The angle estimation performance of the proposed algorithm is much better than that of the conventional Capon algorithm and very close to that of the two-dimensional NC-Capon algorithm, which has a much higher complexity than the proposed algorithm. Furthermore, the proposed algorithm can be applied to arbitrary arrays and works well without estimating the noncircular phases. The simulation results verify the effectiveness and improvement of the proposed algorithm.
\end{abstract}

\section{Introduction}

Direction of arrival (DOA) estimation is a hot topic in the array signal processing field and has been widely used in communication, radar, sonar, and medical image [1-4]. Classical DOA estimation algorithms include multiple signal classification (MUSIC) [5], estimation of signal parameters via rotational invariance technique (ESPRIT) [6-8], propagator method [9], and the Capon [10]. Besides, compressive sensing (CS) [11] and Bayesian compressive sensing (BCS) [12] have recently been used to solve the problem of DOA estimation, and they have an advantage of not requiring knowledge of the number of impinging signals.

To improve the DOA estimation performance, the noncircular property of incoming signals has been considered in [13-22]. In wireless communications, the noncircular signals have been extensively used, for example, the binary phase shift keying, amplitude modulation, and unbalanced quadrature phase shift keying [22]. If $E\{b(t)\}=0, E\left\{b(t) b^{H}(t)\right\} \neq 0$, and $E\left\{b(t) b^{T}(t)\right\} \neq 0$, then $b(t)$ is a noncircular signal [1315]. This statistics redundancy can be properly exploited to enhance the DOA estimation performance. In general, we use the array output and its conjugation to extend the data model and array aperture. A noncircular MUSIC (NC-MUSIC) algorithm was proposed in [14] for the DOA estimation of the noncircular signals. In order to avoid the peak search in NC-MUSIC, a polynomial rooting NC-MUSIC (NC-RootMUSIC) was presented in [15]. NC-ESPRIT algorithms were proposed in $[16,17]$ for DOA estimation without spectrum search. Real-valued implementation of unitary ESPRIT (NCUnitary-ESPRIT) for noncircular sources was presented in [18], and it has a low complexity. Besides, a noncircular propagator method (NC-PM) for direction estimation of noncircular signals was proposed in [19], which has better angle estimation performance than PM in [9]. Based on the parallel factor (PARAFAC) technique, a noncircular PARAFAC (NC-PARAFAC) algorithm was proposed in [20] to obtain the two-dimensional (2D) DOA estimation of the noncircular signals for arbitrarily spaced acoustic vectorsensor array. Moreover, a two-dimensional direction-finding for noncircular signals using two parallel linear arrays via the extended rank reduction algorithm was presented in [21]. 
Many DOA estimation algorithms mentioned above require the prior knowledge of the number of sources or need to estimate the number of sources by information theory algorithm, matrix decomposition algorithm, smoothed rank algorithm, or Gerschgorin disks algorithm [23-26]. Notably, the Capon method can work well without information of the number of sources [10]. CS method and BCS method work well without estimating the number of the sources, but the noncircular property is not considered. By combining the Capon method and the noncircular property, a noncircular Capon (NC-Capon) algorithm was proposed in [27] for the DOA estimation of noncircular signals, but it needs an exhaustive two-dimensional (2D) search over the regions of both DOA and noncircular phase.

In this work, we will propose a reduced-dimension NCCapon (RD-NC-Capon) algorithm for the DOA estimation of noncircular signals. The proposed algorithm, which only requires one-dimensional search, can avoid the high computational cost within the two-dimensional NC-Capon (2D-NC-Capon) algorithm [27]. The angle estimation performance of the proposed algorithm is much better than that of the conventional Capon algorithm and very close to that of the two-dimensional NC-Capon algorithm. Moreover, the proposed algorithm can be applied to arbitrary arrays and works well without estimating the noncircular phases. Numerical simulations verify the improvement and effectiveness of the proposed algorithm.

The remainder of this paper is structured as follows. Section 2 introduces the data model. Section 3 proposes the RD-NC-Capon algorithm. Section 4 gives the performance analysis. In Section 5, simulation results are provided to show the effectiveness, while the conclusions are drawn in Section 6.

Notations. Lowercase (capital) bold symbols denote vector (matrix). $(\cdot)^{*},(\cdot)^{T},(\cdot)^{H},(\cdot)^{-1}$, and $(\cdot)^{+}$denote complex conjugation, transpose, conjugate-transpose, inverse, and pseudoinverse operations, respectively. $\operatorname{diag}\{\mathbf{v}\}$ stands for a diagonal matrix whose diagonal is a vector $\mathbf{v}$. $\operatorname{Re}(\cdot)$ is to get the real part of the complex. $E\{\cdot\}$ presents the statistical expectation. $[\mathbf{P}]_{i, j}$ denotes the $(i, j)$ element of the matrix $\mathbf{P} . \oplus$ is Hadamard product. $\mathbf{I}_{M}$ stands for an $M \times M$ identity matrix and $\mathbf{0}_{M \times N}$ is a zero matrix with $M \times N$.

\section{Data Model}

As shown in Figure 1, we consider a linear array consisting of $M$ omnidirectional sensors and select the first sensor as the referenced one. The vector $\mathbf{d}=\left[d_{1}, d_{2}, \ldots, d_{M}\right]$ denotes the displacement between the sensor and the referenced one, and $d_{1}=0$. We assume that there are $K$ far-field, narrow-band signals impinging on the linear array from different angles $\theta_{k}(k=1,2, \ldots, K)$. The received signal of the array can be expressed by vector $\mathbf{x}(t)[28]$ :

$$
\mathbf{x}(t)=\mathbf{A s}(t)+\mathbf{n}(t),
$$

where $\mathbf{A}=\left[\mathbf{a}\left(\theta_{1}\right), \ldots, \mathbf{a}\left(\theta_{K}\right)\right] \in \mathbb{C}^{M \times K}$ with $\mathbf{a}\left(\theta_{k}\right)=$ $\left[1, e^{-j 2 \pi d_{2} \sin \theta_{k} / \lambda}, \ldots, e^{-j 2 \pi d_{M} \sin \theta_{k} / \lambda}\right]^{T} \in \mathbb{C}^{M \times 1}$ and $\lambda$ being

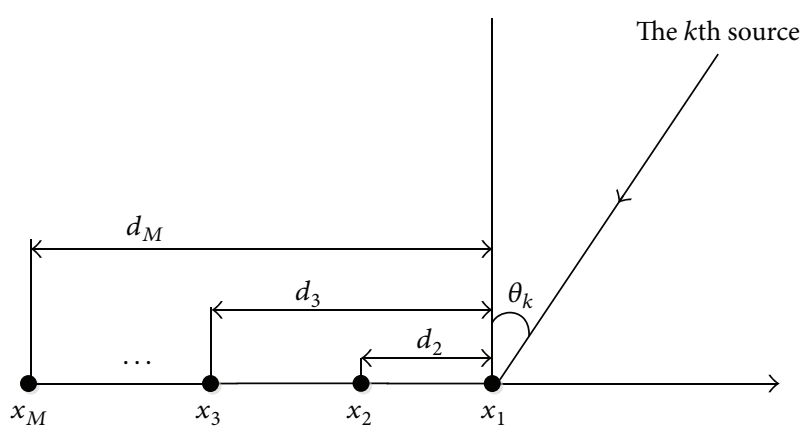

FIgURE 1: Array geometry.

the wavelength. $\mathbf{s}(t) \in \mathbb{C}^{K \times 1}$ is the narrow-band noncircular signal vector and $\mathbf{n}(t)$ denotes the additive white Gaussian noise.

As for the definition of noncircular signal, we consider that if $E\{\mathbf{s}(t)\}=\mathbf{0}_{K \times 1}, E\left\{\mathbf{s}(t) \mathbf{s}^{H}(t)\right\} \neq \mathbf{0}_{K \times K}$, and $E\left\{\mathbf{s}(t) \mathbf{s}^{T}(t)\right\}=$ $\mathbf{0}_{K \times K}$, then $\mathbf{s}(t)$ is a circular signal vector. Otherwise, if $E\{\mathbf{s}(t)\}=\mathbf{0}_{K \times 1}, E\left\{\mathbf{s}(t) \mathbf{s}^{H}(t)\right\} \neq \mathbf{0}_{K \times K}$, and $E\left\{\mathbf{s}(t) \mathbf{s}^{T}(t)\right\} \neq$ $\mathbf{0}_{K \times K}$, then $\mathbf{s}(t)$ is a noncircular signal vector [13-19]. Additionally, the vector of strictly second-order noncircular signals can be expressed as [13-16]

$$
\mathbf{s}(t)=\Psi \mathbf{s}_{0}(t)
$$

where $\mathbf{s}_{0}(t) \in \mathbb{R}^{K \times 1}$ and $\Psi=\operatorname{diag}\left\{e^{-j \phi_{1}}, e^{-j \phi_{2}}, \ldots, e^{-j \phi_{K}}\right\}$ with $\phi_{k}$ being the noncircular phase of the $k$ th signal. According to (2), we rewrite (1) as

$$
\mathbf{x}(t)=\mathbf{A} \Psi \mathbf{s}_{0}(t)+\mathbf{n}(t) .
$$

\section{DOA Estimation Algorithm}

In this section, we will propose the RD-NC-Capon algorithm for the DOA estimation of noncircular signals. In order to double the array aperture, we will first give the extended data model by exploiting the noncircular property, which is a common method of expansion for noncircular signals and has been widely used in many noncircular DOA estimation algorithms [13-22]. Then we will talk about the conventional 2D-NC-Capon algorithm in Section 3.2, whereas the RDNC-Capon algorithm will be proposed in Section 3.3.

3.1. Data Construction. When the noncircular signals impinge on the array, we use the array output and its conjugation to extend the data model [13-15]:

$$
\begin{aligned}
\mathbf{y}(t) & =\left[\begin{array}{c}
\mathbf{x}(t) \\
\mathbf{x}^{*}(t)
\end{array}\right]=\left[\begin{array}{c}
\mathbf{A} \boldsymbol{\Psi} \\
\mathbf{A}^{*} \Psi^{*}
\end{array}\right] \mathbf{s}_{0}(t)+\left[\begin{array}{c}
\mathbf{n}(t) \\
\mathbf{n}^{*}(t)
\end{array}\right] \\
& =\mathbf{B s}_{0}(t)+\mathbf{n}_{0}(t)
\end{aligned}
$$


where $\mathbf{n}_{0}(t)=\left[\begin{array}{c}\mathbf{n}(t) \\ \mathbf{n}^{*}(t)\end{array}\right] \in \mathbb{C}^{2 M \times 1}$. Consider $\mathbf{B}=\left[\begin{array}{c}\mathbf{A} \Psi \\ \mathbf{A}^{*} \Psi^{*}\end{array}\right] \in$ $\mathbb{C}^{2 M \times K}$, and it can be written as

B

$$
=\left[\begin{array}{cccc}
e^{-j \phi_{1}} & e^{-j \phi_{2}} & \cdots & e^{-j \phi_{K}} \\
e^{-j\left(2 \pi d_{2} \sin \theta_{1} / \lambda+\phi_{1}\right)} & e^{-j\left(2 \pi d_{2} \sin \theta_{2} / \lambda+\phi_{2}\right)} & \cdots & e^{-j\left(2 \pi d_{2} \sin \theta_{K} / \lambda+\phi_{K}\right)} \\
\vdots & \vdots & \ddots & \vdots \\
e^{-j\left(2 \pi d_{M} \sin \theta_{1} / \lambda+\phi_{1}\right)} & e^{-j\left(2 \pi d_{M} \sin \theta_{2} / \lambda+\phi_{2}\right)} & \cdots & e^{-j\left(2 \pi d_{M} \sin \theta_{K} / \lambda+\phi_{K}\right)} \\
e^{j \phi_{1}} & e^{j \phi_{2}} & \cdots & e^{j \phi_{K}} \\
e^{j\left(2 \pi d_{2} \sin \theta_{1} / \lambda+\phi_{1}\right)} & e^{j\left(2 \pi d_{2} \sin \theta_{2} / \lambda+\phi_{2}\right)} & \cdots & e^{j\left(2 \pi d_{2} \sin \theta_{K} / \lambda+\phi_{K}\right)} \\
\vdots & \vdots & \ddots & \vdots \\
e^{j\left(2 \pi d_{M} \sin \theta_{1} / \lambda+\phi_{1}\right)} & e^{j\left(2 \pi d_{M} \sin \theta_{2} / \lambda+\phi_{2}\right)} & \cdots & e^{j\left(2 \pi d_{M} \sin \theta_{K} / \lambda+\phi_{K}\right)}
\end{array}\right] .
$$

The covariance matrix of the extended data model can be expressed as $\mathbf{R}=E\left[\mathbf{y}(t) \mathbf{y}^{H}(t)\right]$.

3.2. 2D-NC-Capon Algorithm. The following 2D-NC-Capon function can be utilized to estimate the DOAs of the noncircular signals $[27,28]$

$$
P_{\text {2D-NC-Capon }}(\theta, \phi)=\frac{1}{\mathbf{b}^{H}(\theta, \phi) \mathbf{R}^{-1} \mathbf{b}(\theta, \phi)},
$$

where

$$
\mathbf{b}(\theta, \phi)=\left[\begin{array}{c}
e^{-j \phi} \\
e^{-j\left(2 \pi d_{2} \sin \theta / \lambda+\phi\right)} \\
\vdots \\
e^{-j\left(2 \pi d_{M} \sin \theta / \lambda+\phi\right)} \\
e^{j \phi} \\
e^{j\left(2 \pi d_{2} \sin \theta / \lambda+\phi\right)} \\
\vdots \\
e^{j\left(2 \pi d_{M} \sin \theta / \lambda+\phi\right)}
\end{array}\right] .
$$

The 2D-NC-Capon algorithm can obtain $K$ local peak values of (6) by 2D search of $\theta$ and $\phi$. Since the 2D-NC-Capon algorithm requires an exhaustive $2 \mathrm{D}$ search, this approach is normally inefficient due to its high computational cost. A reduced-dimension (RD) Capon algorithm was proposed in [29] for the angle estimation of bistatic multiple-input multiple-output radar. In the following subsections, we make reference to the RD idea from [29] and present a RD-NCCapon algorithm for the DOA estimation of noncircular signals just through one-dimensional (1D) search.

There are some differences between the work in [29] and our work. Firstly, [29] used the RD-Capon method for angle estimation in bistatic multiple-input multiple-output radar, while our work is to extend the RD-Capon idea and propose RD-NC-Capon for angle estimation of noncircular signals. Secondly, the noncircular property is not considered in [29], and we use the noncircular property of the sources to double the array aperture and enhance the angle estimation performance. Thirdly, the modeling in each work is different. The received signal in [26] can be used directly for $\mathrm{RD}$ processing, while a data model extension is required in our work.

3.3. The Proposed RD-NC-Capon Algorithm. Equation (7) can be denoted by

$$
\begin{aligned}
\mathbf{b}(\theta, \phi) & =\left[\begin{array}{l}
\mathbf{a}(\theta) e^{-j \phi} \\
\mathbf{a}^{*}(\theta) e^{j \phi}
\end{array}\right]=\left[\begin{array}{ll}
\mathbf{a}(\theta) & \mathbf{0}_{M \times 1} \\
\mathbf{0}_{M \times 1} & \mathbf{a}^{*}(\theta)
\end{array}\right]\left[\begin{array}{c}
e^{-j \phi} \\
e^{j \phi}
\end{array}\right] \\
& =\mathbf{P}(\theta) \mathbf{e}_{0}(f)
\end{aligned}
$$

where $\mathbf{0}_{M \times 1}$ is an $M \times 1$ zero matrix. Consider

$$
\begin{aligned}
\mathbf{a}(\theta) & =\left[1, e^{-j 2 \pi d_{2} \sin \theta / \lambda}, \ldots, e^{-j 2 \pi d_{M} \sin \theta / \lambda}\right]^{T}, \\
\mathbf{P}(\theta) & =\left[\begin{array}{ll}
\mathbf{a}(\theta) & \mathbf{0}_{M \times 1} \\
\mathbf{0}_{M \times 1} & \mathbf{a}^{*}(\theta)
\end{array}\right], \\
\mathbf{e}_{0}(\phi) & =\left[\begin{array}{c}
e^{-j \phi} \\
e^{j \phi}
\end{array}\right] .
\end{aligned}
$$

Then we construct the following function:

$$
V(\theta, \phi)=\frac{1}{P_{2 \text { D-NC-Capon }}(\theta, \phi)} .
$$

According to (6)-(10), we know that

$$
V(\theta, \phi)=\mathbf{e}_{0}^{H}(\phi) \mathbf{P}^{H}(\theta) \mathbf{R}^{-1} \mathbf{P}(\theta) \mathbf{e}_{\mathbf{0}}(\phi) .
$$

Obviously, $V(\theta, \phi)$ can be expressed as

$$
V(\theta, \phi)=e^{-j \phi} e^{j \phi} \mathbf{e}_{0}^{H}(\phi) \mathbf{P}^{H}(\theta) \mathbf{R}^{-1} \mathbf{P}(\theta) \mathbf{e}_{\mathbf{0}}(\phi) .
$$

Equation (12) can be denoted by

$$
V(\theta, \phi)=e^{-j \phi} \mathbf{e}_{0}^{H}(\phi) \mathbf{P}^{H}(\theta) \mathbf{e}_{\mathbf{0}}(\phi) e^{j \phi} .
$$

We define $\mathbf{q}(\phi)=\mathbf{e}_{\mathbf{0}}(\phi) e^{j \phi}=\left[\begin{array}{c}1 \\ e^{j 2 \phi}\end{array}\right]$ and $\mathbf{Q}(\theta)=$ $\mathbf{P}^{H}(\theta) \mathbf{R}^{-1} \mathbf{P}(\theta)$. Equation (13) can be rewritten as

$$
V(\theta, \phi)=\mathbf{q}^{H}(\phi) \mathbf{Q}(\theta) \mathbf{q}(\phi) .
$$

As (14) is a quadratic optimization, we consider eliminating trivial solution $\mathbf{q}(\phi)=\mathbf{0}_{2 \times 1}$ and add a constraint of $\mathbf{e}^{H} \mathbf{q}(\phi)=$ 1 , where $\mathbf{e}=[1,0]^{T}$. Then the optimization problem in (14) is reconstructed as follows:

$$
\begin{array}{ll}
\min _{\theta, \phi} & \mathbf{q}^{H}(\phi) \mathbf{Q}(\theta) \mathbf{q}(\phi) \\
\text { s.t. } & \mathbf{e}^{H} \mathbf{q}(\phi)=1 .
\end{array}
$$

We construct the following cost function using Lagrange multiplier:

$$
L(\theta, \phi)=\mathbf{q}^{H}(\phi) \mathbf{Q}(\theta) \mathbf{q}(\phi)-\rho\left[\mathbf{e}^{H} \mathbf{q}(\phi)-1\right],
$$


where $\rho$ is a constant. If the derivative of (16) is set to zero, that is,

$$
\frac{\partial}{\partial \mathbf{q}(\phi)} L(\theta, \phi)=2 \mathbf{Q}(\theta) \mathbf{q}(\phi)+\rho \mathbf{e}=0,
$$

then $\mathbf{q}(\phi)=-0.5 \rho \mathbf{Q}^{-1}(\theta)$ e. We define $\mu=-0.5 \rho$; then we get

$$
\widehat{\mathbf{q}}(\phi)=\mu \mathbf{Q}(\theta)^{-1} \mathbf{e} .
$$

As $\mathbf{e}^{H} \mathbf{q}(\phi)=1$ and $\mu=1 / \mathbf{e}^{H} \mathbf{Q}(\theta)^{-1} \mathbf{e}$, we have

$$
\widehat{\mathbf{q}}(\phi)=\frac{\mathbf{Q}^{-1}(\theta) \mathbf{e}}{\mathbf{e}^{H} \mathbf{Q}^{-1}(\theta) \mathbf{e}} .
$$

From (14) and (19), we have

$$
\widehat{\theta}=\arg \min \frac{1}{\mathbf{e}^{H} \mathbf{Q}^{-1}(\theta) \mathbf{e}}=\arg \max \mathbf{e}^{H} \mathbf{Q}^{-1}(\theta) \mathbf{e} .
$$

As $\mathbf{Q}(\theta)=\mathbf{P}(\theta)^{H} \mathbf{R}^{-1} \mathbf{P}(\theta)$, a new $1 \mathrm{D}$ search cost function is used for DOA estimation:

$$
f_{\text {NC-RD-Capon }}(\theta)=\mathbf{e}^{H}\left(\mathbf{P}(\theta)^{H} \mathbf{R}^{-1} \mathbf{P}(\theta)\right)^{-1} \mathbf{e} .
$$

In the finite sample case, the covariance matrix can be estimated as

$$
\widehat{\mathbf{R}}=\frac{1}{L} \sum_{l=1}^{L} \mathbf{y}\left(t_{l}\right) \mathbf{y}^{H}\left(t_{l}\right)
$$

where $L$ denotes the number of snapshots. Then, the DOAs $\theta_{k}(k=1,2, \ldots, K)$ can be obtained through the following search:

$$
f_{\text {RD-NC-Capon }}(\theta)=\mathbf{e}^{H}\left(\mathbf{P}(\theta)^{H} \widehat{\mathbf{R}}^{-1} \mathbf{P}(\theta)\right)^{-1} \mathbf{e} .
$$

2D-NC-Capon algorithm obtains DOA estimation by 2D search of $\theta$ and $\phi$, whereas the proposed algorithm gets DOA estimation via $1 \mathrm{D}$ search according to the function in (23). The proposed RD-NC-Capon algorithm can work well without estimation of noncircular phases.

The main steps of RD-NC-Capon algorithm are shown as follows.

Step 1. Construct the extended data model from the array output via (4).

Step 2. Compute the covariance matrix $\widehat{\mathbf{R}}$ of the extended data model via (22).

Step 3. Use the $1 \mathrm{D}$ spectrum search function to estimate DOAs via (23).

\section{Performance Analysis}

In this section, we first analyze the computational complexity of the proposed algorithm and then derive the Cramér-Rao bound (CRB) of DOA estimation.
4.1. Complexity Analysis. For the proposed algorithm, computing the covariance matrix requires $O\left(4 M^{2} L\right)$, inversion operation of the covariance matrix needs $O\left(8 M^{3}\right)$, and a spectrum searching wants $O\left(8\left(M^{2}+M\right)\right)$. The main computational complexity of the proposed RD-NC-Capon algorithm is $O\left(4 M^{2} L+8 M^{3}+8\left(M^{2}+M\right) n\right)$, where $n$ is the number of searches, whereas the 2D-NC-Capon algorithm needs $O\left(4 M^{2} L+8 M^{3}+\left(4 M^{2}+2 M\right) n^{2}\right)$. Thus, the RD-NC-Capon algorithm has a much lower computational complexity than the 2D-NC-Capon algorithm. Figure 2 shows the complexity comparison versus the snapshots $L$, where $K=3, n=6000$, and $M=8$ are considered. Figure 3 presents the running time comparison, where we compare the proposed algorithm against the 2D-NC-Capon algorithm. From Figures 2 and 3 , we find that the proposed algorithm has a much lower complexity than the 2D-NC-Capon algorithm.

4.2. Cramér-Rao Bound. In the finite sample case, the data model can be rewritten as

$$
\mathbf{Y}=\mathbf{B S}_{0}+\mathbf{N}_{0},
$$

where $\mathbf{Y}=\left[\mathbf{y}\left(t_{1}\right), \ldots, \mathbf{y}\left(t_{L}\right)\right]$ and $\mathbf{S}_{0}=\left[\mathbf{s}_{0}\left(t_{1}\right), \ldots, \mathbf{s}_{0}\left(t_{L}\right)\right] . \mathbf{B} \in$ $\mathbb{C}^{2 M \times K}$ is the same as that shown in (5).

We assume that the signal is deterministic, and then the estimation parameter vector is expressed as

$$
\zeta=\left[\theta_{1}, \ldots, \theta_{K}, \phi_{1}, \ldots, \phi_{K}, \mathbf{s}_{0}^{T}\left(t_{1}\right), \ldots, \mathbf{s}_{0}^{T}\left(t_{L}\right), \sigma^{2}\right]^{T},
$$

where $\mathbf{s}_{0}^{T}\left(t_{l}\right)$ is the $l$ th column of $\mathbf{S}_{0} . \sigma^{2}$ is the noise power.

According to (24), we have

$$
\mathbf{y}=\operatorname{vec}(\mathbf{Y})=\operatorname{vec}\left(\mathbf{B S}_{0}\right)+\operatorname{vec}\left(\mathbf{N}_{0}\right),
$$

where $\operatorname{vec}(\cdot)$ is to convert a matrix into a vector.

The expected value $\boldsymbol{\mu}$ and the covariance matrix $\Gamma$ of $y$ are

$$
\begin{aligned}
& \boldsymbol{\mu}=\left[\begin{array}{c}
\mathbf{B s}\left(t_{1}\right) \\
\vdots \\
\mathbf{B s}\left(t_{L}\right)
\end{array}\right]=\mathbf{G S}, \\
& \boldsymbol{\Gamma}=\left[\begin{array}{ccc}
\sigma^{2} \mathbf{I}_{2 M} & & \mathbf{0} \\
& \ddots & \\
\mathbf{0} & & \sigma^{2} \mathbf{I}_{2 M}
\end{array}\right],
\end{aligned}
$$

where

$$
\mathbf{G}=\left[\begin{array}{lll}
\mathbf{B} & & \mathbf{0} \\
& \ddots & \\
\mathbf{0} & & \mathbf{B}
\end{array}\right],
$$

$$
\mathbf{S}=\left[\begin{array}{c}
\mathbf{s}_{0}\left(t_{1}\right) \\
\vdots \\
\mathbf{s}_{0}\left(t_{L}\right)
\end{array}\right] \text {. }
$$




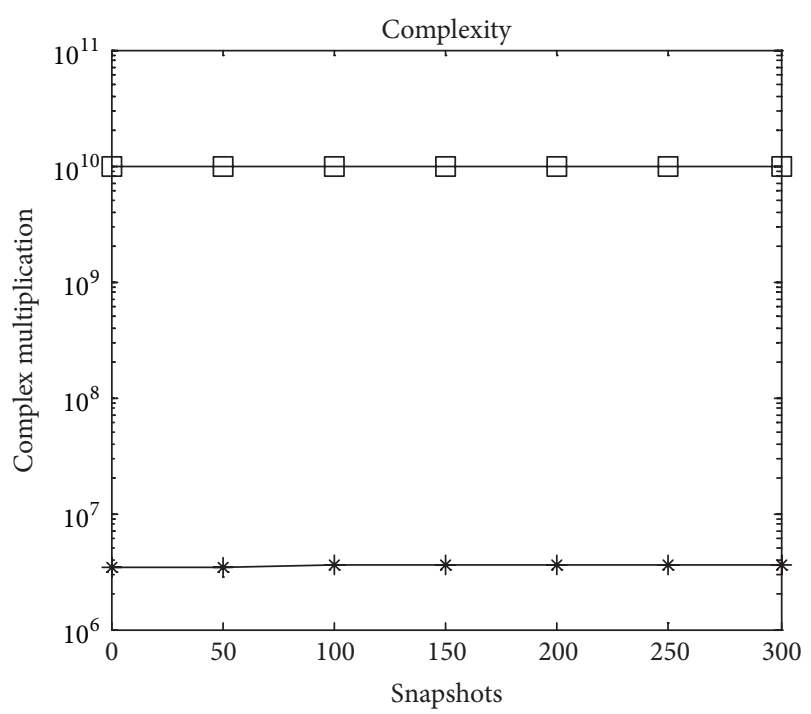

$\square$ 2D-NC-Capon

* RD-NC-Capon

FIGURE 2: The complexity comparison.

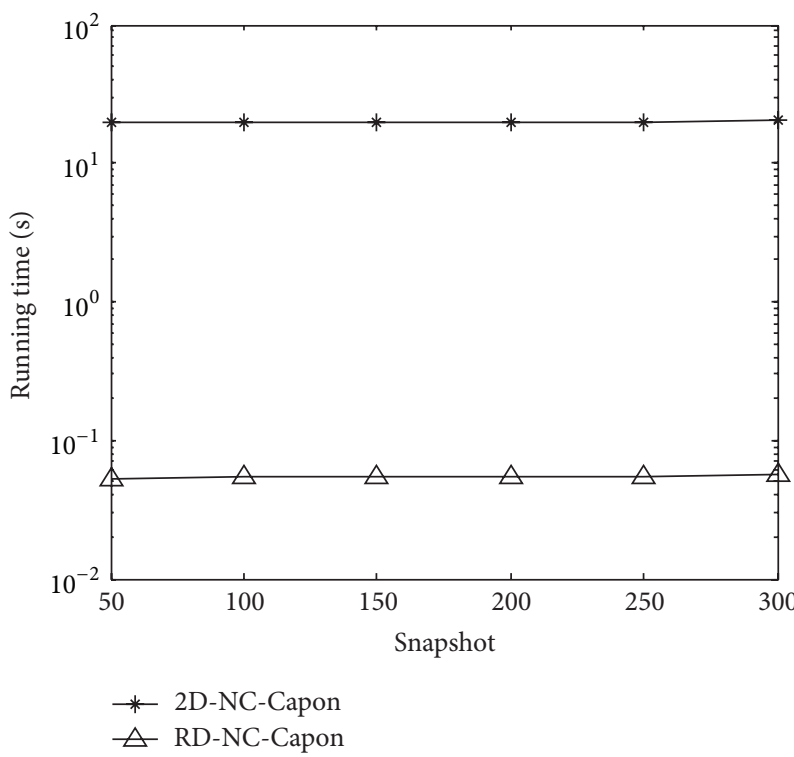

FIGURE 3: The running time comparison.

According to $[22,30,31]$, we know that the $(i, j)$ element of the CRB matrix $\left(\mathbf{P}_{\text {cr }}\right)$ can be expressed as

$$
\left[\mathbf{P}_{\mathrm{cr}}^{-1}\right]_{i j}=\operatorname{tr}\left[\boldsymbol{\Gamma}^{-1} \boldsymbol{\Gamma}_{i}^{\prime} \boldsymbol{\Gamma}^{-1} \boldsymbol{\Gamma}_{j}^{\prime}\right]+2 \operatorname{Re}\left[\boldsymbol{\mu}_{i}^{\prime H} \boldsymbol{\Gamma}^{-1} \boldsymbol{\mu}_{j}^{\prime}\right],
$$

where $\boldsymbol{\Gamma}_{i}^{\prime}, \boldsymbol{\Gamma}_{j}^{\prime}$ and $\boldsymbol{\mu}_{i}^{\prime}, \boldsymbol{\mu}_{j}^{\prime}$ are the derivatives of $\boldsymbol{\Gamma}$ and $\boldsymbol{\mu}$ on the $i$ th or $j$ th element of $\zeta$, respectively. $\operatorname{tr}[\cdot]$ is to obtain the trace of a matrix. Since the covariance matrix is just related to $\sigma^{2}$, the first part of (29) is zero. Then

$$
\left[\mathbf{P}_{\mathrm{cr}}^{-\mathbf{1}}\right]_{i j}=2 \operatorname{Re}\left[\boldsymbol{\mu}_{i}^{\prime H} \boldsymbol{\Gamma}^{-1} \boldsymbol{\mu}_{j}^{\prime}\right] .
$$

We have

$$
\begin{gathered}
\frac{\partial \boldsymbol{\mu}}{\partial \theta_{k}}=\left[\begin{array}{c}
\frac{\partial \mathbf{B}}{\partial \theta_{k}} \mathbf{s}_{0}\left(t_{1}\right) \\
\vdots \\
\frac{\partial \mathbf{B}}{\partial \theta_{k}} \mathbf{s}_{0}\left(t_{L}\right)
\end{array}\right]=\left[\begin{array}{c}
\mathbf{d}_{k \theta} s_{0 k}\left(t_{1}\right) \\
\vdots \\
\mathbf{d}_{k \theta} s_{0 k}\left(t_{L}\right)
\end{array}\right], \\
\frac{\partial \boldsymbol{\mu}}{\partial \phi_{k}}=\left[\begin{array}{c}
\frac{\partial \mathbf{B}}{\partial \phi_{k}} \mathbf{s}_{0}\left(t_{1}\right) \\
\vdots \\
\frac{\partial \mathbf{B}}{\partial \phi_{k}} \mathbf{s}_{0}\left(t_{L}\right)
\end{array}\right]=\left[\begin{array}{c}
\mathbf{d}_{k \phi} s_{0 k}\left(t_{1}\right) \\
\vdots \\
\mathbf{d}_{k \phi} s_{0 k}\left(t_{L}\right)
\end{array}\right], \quad \begin{array}{l}
k=1, \ldots, K, \\
k=1,
\end{array}
\end{gathered}
$$

where $s_{0 k}(t)$ is the $k$ th element of $\mathbf{s}_{0}(t), \mathbf{d}_{k \theta}=\partial \mathbf{b}_{k} / \partial \theta_{k}$, and $\mathbf{d}_{k \phi}=\partial \mathbf{b}_{k} / \partial \phi_{k}$ with $\mathbf{b}_{k}$ being the $k$ th column of $\mathbf{B}$.

We define

$\Delta$

$$
=\left[\begin{array}{cccccc}
\mathbf{d}_{1 \theta} s_{01}\left(t_{1}\right) & \cdots & \mathbf{d}_{K \theta} s_{0 K}\left(t_{1}\right) & \mathbf{d}_{1 \phi} s_{01}\left(t_{1}\right) & \cdots & \mathbf{d}_{K \phi} s_{0 K}\left(t_{1}\right) \\
\vdots & & \vdots & \vdots & & \vdots \\
\mathbf{d}_{1 \theta} s_{01}\left(t_{L}\right) & \cdots & \mathbf{d}_{K \theta} s_{0 K}\left(t_{L}\right) & \mathbf{d}_{1 \phi} s_{01}\left(t_{L}\right) & \cdots & \mathbf{d}_{K \phi} s_{0 K}\left(t_{L}\right)
\end{array}\right]
$$

As $\boldsymbol{\mu}=\mathbf{G S}$,

$$
\frac{\partial \boldsymbol{\mu}}{\partial \mathbf{S}^{T}}=\mathbf{G}
$$

Now we have

$$
\frac{\partial \boldsymbol{\mu}}{\partial \zeta^{T}}=\left[\Delta, \mathbf{G}, \mathbf{0}_{2 M L \times 1}\right] .
$$

According to (34),

$$
2 \operatorname{Re}\left\{\frac{\partial \boldsymbol{\mu}^{H}}{\partial \boldsymbol{\zeta}} \boldsymbol{\Gamma}^{-1} \frac{\partial \boldsymbol{\mu}}{\partial \boldsymbol{\zeta}^{T}}\right\}=\left[\begin{array}{cc}
\mathbf{J} & \mathbf{0}_{(K L+2 K) \times 1} \\
\mathbf{0}_{1 \times(K L+2 K)} & 0
\end{array}\right],
$$

where $\mathbf{J}=\left(2 / \sigma^{2}\right) \operatorname{Re}\left\{\left[\begin{array}{c}\Delta^{H} \\ \mathbf{G}^{H}\end{array}\right]\left[\begin{array}{ll}\Delta & \mathbf{G}\end{array}\right]\right\}$.

Define

$$
\begin{gathered}
\mathbf{Q}=\left(\mathbf{G}^{H} \mathbf{G}\right)^{-1} \mathbf{G}^{H} \Delta, \\
\mathbf{F}=\left[\begin{array}{cc}
\mathbf{I}_{2 K} & \mathbf{0}_{2 K \times K L} \\
-\mathbf{Q} & \mathbf{I}_{K L}
\end{array}\right],
\end{gathered}
$$

where $\mathbf{Q}_{R}$ and $\mathbf{Q}_{I}$ are the real and imaginary parts of $\mathbf{Q}$, respectively.

It can be demonstrated that

$$
\left[\begin{array}{ll}
\Delta & \mathbf{G}
\end{array}\right] \mathbf{F}=\left[\begin{array}{ll}
(\Delta-\mathbf{G Q}) & \mathbf{G}
\end{array}\right]=\left[\Pi_{\mathbf{G}}^{\perp} \Delta \mathrm{G}\right],
$$

where $\boldsymbol{\Pi}_{\mathbf{G}}^{\perp}=\mathbf{I}-\mathbf{G}\left(\mathbf{G}^{H} \mathbf{G}\right)^{-1} \mathbf{G}^{H}$ and $\mathbf{G}^{H} \boldsymbol{\Pi}_{\mathbf{G}}^{\perp}=\mathbf{0}$. Then we have

$$
\mathbf{F}^{T} \mathbf{J F}=\frac{2}{\sigma^{2}} \operatorname{Re}\left\{\mathbf{F}^{H}\left[\begin{array}{l}
\Delta^{H} \\
\mathbf{G}^{H}
\end{array}\right]\left[\begin{array}{ll}
\Delta & \mathbf{G}
\end{array}\right] \mathbf{F}\right\} .
$$


According to (37), we know that

$$
\begin{aligned}
\mathbf{F}^{H}\left[\begin{array}{c}
\Delta^{H} \\
\mathbf{G}^{H}
\end{array}\right]\left[\begin{array}{ll}
\Delta & \mathbf{G}
\end{array}\right] \mathbf{F} & =\left[\begin{array}{c}
\Delta^{H} \boldsymbol{\Pi}_{\mathbf{G}}^{\perp} \\
\mathbf{G}^{H}
\end{array}\right]\left[\begin{array}{ll}
\boldsymbol{\Pi}_{\mathbf{G}}^{\perp} & \mathbf{G}
\end{array}\right] \\
& =\left[\begin{array}{cc}
\Delta^{H} \boldsymbol{\Pi}_{\mathbf{G}}^{\perp} & \mathbf{0}_{2 K \times K L} \\
\mathbf{0}_{K L \times 2 K} & \mathbf{G}^{H} \mathbf{G}
\end{array}\right] .
\end{aligned}
$$

Hence, combining (37)-(38), $\mathbf{F}^{T} \mathbf{J F}$ can be rewritten as

$$
\mathbf{F}^{T} \mathbf{J F}=\frac{2}{\sigma^{2}} \operatorname{Re}\left\{\left[\begin{array}{cc}
\Delta^{H} \boldsymbol{\Pi}_{\mathbf{G}}^{\perp} \boldsymbol{\Delta} & \mathbf{0}_{2 K \times K L} \\
\mathbf{0}_{K L \times 2 K} & \mathbf{G}^{H} \mathbf{G}
\end{array}\right]\right\} .
$$

It is obvious that $\mathbf{J}^{-1}=\mathbf{F}\left(\mathbf{F}^{T} \mathbf{J F}\right)^{-1} \mathbf{F}^{T}$. Then $\mathbf{J}^{-1}$ is written as

$$
\begin{aligned}
\mathbf{J}^{-1}= & \frac{\sigma^{2}}{2}\left[\begin{array}{cc}
\mathbf{I}_{2 K} & \mathbf{0}_{2 K \times K L} \\
-\mathbf{Q} & \mathbf{I}_{K L}
\end{array}\right]\left[\begin{array}{cc}
\operatorname{Re}\left(\Delta^{H} \Pi_{\mathbf{G}}^{\perp} \boldsymbol{\Delta}\right) & \mathbf{0}_{2 K \times K L} \\
\mathbf{0}_{K L \times 2 K} & \mathbf{G}^{H} \mathbf{G}
\end{array}\right]^{-1} \\
& \cdot\left[\begin{array}{cc}
\mathbf{I}_{2 K} & -\mathbf{Q}^{T} \\
\mathbf{0}_{2 K \times K L} & \mathbf{I}_{K L}
\end{array}\right] .
\end{aligned}
$$

Furthermore,

$$
\mathbf{J}^{-1}=\left[\begin{array}{cc}
\frac{\sigma^{2}}{2}\left[\operatorname{Re}\left(\Delta^{H} \Pi_{\mathbf{G}}^{\perp} \Delta\right)\right]^{-1} & \kappa \\
\kappa & \kappa
\end{array}\right],
$$

where $\kappa$ denotes the part we are not concerned about.

Till now, we can give the CRB matrix [30, 31]:

$$
\mathrm{CRB}=\frac{\sigma^{2}}{2}\left[\operatorname{Re}\left(\Delta^{H} \Pi_{\mathbf{G}}^{\perp} \Delta\right)\right]^{-1} .
$$

After further simplification, we rewrite the CRB matrix as

$$
\mathrm{CRB}=\frac{\sigma^{2}}{2 L}\left\{\operatorname{Re}\left[\mathbf{D}^{H} \boldsymbol{\Pi}_{\mathbf{B}}^{\perp} \mathbf{D} \oplus \widehat{\mathbf{R}}_{s}\right]\right\}^{-1},
$$

where $\sigma^{2}$ is noise power, $\widehat{\mathbf{R}}_{s}=(1 / L) \sum_{l=1}^{L} \mathbf{s}_{0}\left(t_{l}\right) \mathbf{s}_{0}{ }^{H}\left(t_{l}\right)$, and $\boldsymbol{\Pi}_{\mathbf{B}}^{\perp}=\mathbf{I}_{2 M}-\mathbf{B}\left(\mathbf{B}^{H} \mathbf{B}\right)^{-1} \mathbf{B}^{H}$. Consider $\mathbf{D}=\left[\mathbf{d}_{1 \theta}, \mathbf{d}_{2 \theta}, \ldots, \mathbf{d}_{K \theta}\right.$, $\left.\mathbf{d}_{1 \phi}, \mathbf{d}_{2 \phi}, \ldots, \mathbf{d}_{K \phi}\right], \mathbf{d}_{k \theta}=\partial \mathbf{b}_{k} / \partial \theta_{k}$, and $\mathbf{d}_{k \phi}=\partial \mathbf{b}_{k} / \partial \phi_{k}$ with $\mathbf{b}_{k}$ being the $k$ th column of $\mathbf{B}$.

\subsection{Discussions}

Remark 1 . The strictly second-order noncircular signals are considered in the paper. The proposed algorithm works well for the general noncircular signals. When we consider the general noncircular signals, the source vector can be denoted by

$$
\begin{aligned}
\mathbf{s}(t) & =\operatorname{diag}\left(\rho_{1} e^{-j \phi_{1}}, \rho_{2} e^{-j \phi_{2}}, \ldots, \rho_{K} e^{-j \phi_{K}}\right) \mathbf{s}_{0}(t) \\
& =\boldsymbol{\Psi} \boldsymbol{\rho} \mathbf{s}_{0}(t),
\end{aligned}
$$

where $\mathbf{s}_{0}(t) \in \mathbb{R}^{K \times 1}, \boldsymbol{\Psi}=\operatorname{diag}\left\{e^{-j \phi_{1}}, e^{-j \phi_{2}}, \ldots, e^{-j \phi_{K}}\right\}$, and $\boldsymbol{\rho}=$ $\operatorname{diag}\left(\rho_{1}, \rho_{2}, \ldots, \rho_{K}\right) \in \mathbb{R}^{K \times K}$ with $\rho_{k}$ being the noncircularity coefficient of the $k$ th source. The extended signal is

$$
\mathbf{y}(t)=\left[\begin{array}{c}
\mathbf{x}(t) \\
\mathbf{x}^{*}(t)
\end{array}\right]=\mathbf{B} \rho \mathbf{s}_{0}(t)+\mathbf{n}_{0}(t)
$$

We also estimate DOAs of the general noncircular signals in a similar way.

Remark 2. The proposed algorithm works well for the uniform circular array (UCA). We assume $\mathbf{a}(\theta, \varphi)$ is the direction vector of the UCA with DOA being $(\theta, \varphi)$. 3D-NC-Capon function can be utilized to estimate the 2D-DOA of the noncircular signals for UCA. 2D-DOA estimation function of the proposed algorithm is

$$
f_{\text {NC-RD-Capon }}(\theta, \varphi)=\mathbf{e}^{H}\left(\mathbf{P}(\theta, \varphi)^{H} \widehat{\mathbf{R}}^{-1} \mathbf{P}(\theta, \varphi)\right)^{-1} \mathbf{e},
$$

where

$$
\mathbf{P}(\theta, \varphi)=\left[\begin{array}{cc}
\mathbf{a}(\theta, \varphi) & \mathbf{0}_{M \times 1} \\
\mathbf{0}_{M \times 1} & \mathbf{a}^{*}(\theta, \varphi)
\end{array}\right]
$$

\section{Simulation Results}

This section uses Monte Carlo simulations to assess the DOA estimation performance of the proposed algorithm. We define the root mean square error (RMSE) of DOA as

$$
\mathrm{RMSE}=\frac{1}{K} \sum_{k=1}^{K} \sqrt{\frac{1}{1000} \sum_{n=1}^{1000}\left[\widehat{\theta}_{k, n}-\theta_{k}\right]^{2}}
$$

with $\theta_{k}$ being the accurate angle of the $k$ th signal and $\widehat{\theta}_{k, n}$ being the estimate of $\theta_{k}$ of the $n$th Monte Carlo trial. For Figures 4 and $6-9$, we assume that there are $3(K=3)$ noncircular signals impinging on the array with angles being $\left[10^{\circ}, 20^{\circ}, 30^{\circ}\right]$ and the noncircular phases being $\left[10^{\circ}, 30^{\circ}, 50^{\circ}\right]$.

Figure 4 shows the spectrum search result of the proposed algorithm for the nonuniform linear array with $\mathbf{d}=$ $[0,0.45 \lambda, 0.9 \lambda, 1.3 \lambda, 1.78 \lambda, 2.2 \lambda, 2.64 \lambda, 3.1 \lambda]$, where $\lambda$ is the wavelength. In this simulation, signal-to-noise ratio $(\mathrm{SNR})=$ $20 \mathrm{~dB}, M=8$, and $L=200$ are considered. From Figure 4, we find that the proposed algorithm can work well.

Figure 5 presents the spectrum search result of the proposed algorithm for the nonuniform linear array with $\mathbf{d}=[0,0.45 \lambda, 0.9 \lambda, 1.3 \lambda, 1.78 \lambda, 2.2 \lambda, 2.64 \lambda, 3.1 \lambda]$. There are $9(K=9)$ noncircular signals impinging on array with angles being $\left[-40^{\circ},-30^{\circ},-20^{\circ},-10^{\circ}, 0^{\circ}, 10^{\circ}, 20^{\circ}, 30^{\circ}, 40^{\circ}\right]$ and the noncircular phases being $\left[-40^{\circ},-30^{\circ},-20^{\circ},-10^{\circ}, 0^{\circ}, 10^{\circ}\right.$, $\left.20^{\circ}, 30^{\circ}, 40^{\circ}\right]$. SNR $=20 \mathrm{~dB}, M=8$, and $L=200$ are adopted for the simulation. From Figure 5, we find that proposed algorithm works well even when there are more sources than sensors, while the conventional Capon algorithm fails to work in this condition. The Capon algorithm with $M$ elements can identify $M-1$ impinging signals. The proposed algorithm uses the noncircular property to double the number of resolvable sources.

We compare the proposed algorithm against other DOA estimation algorithms. Figure 6 indicates the DOA estimation performance comparison among the Capon algorithm, the 2D-NC-Capon algorithm, and the proposed algorithm with $\mathbf{d}=[0,0.45 \lambda, 0.9 \lambda, 1.3 \lambda, 1.78 \lambda, 2.2 \lambda, 2.64 \lambda, 3.1 \lambda], M=$ $8, L=200$, and $K=3$. ESPRIT and NC-ESPRIT cannot work for nonuniform linear array, because they require 


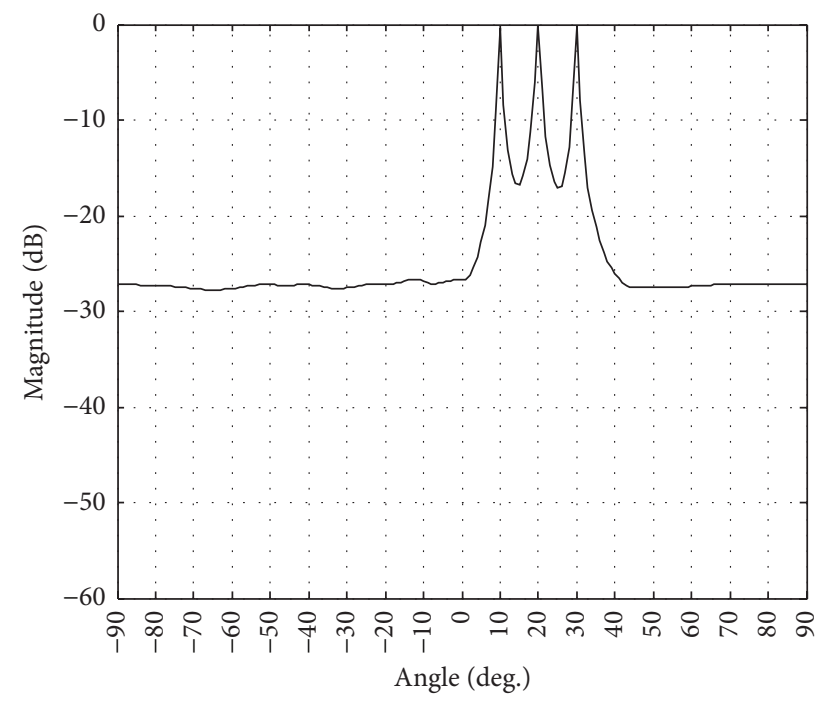

FIGURE 4: The spectrum search results of RD-NC-Capon algorithm $(\mathrm{SNR}=20 \mathrm{~dB}, M=8, L=200$, and $K=3)$.

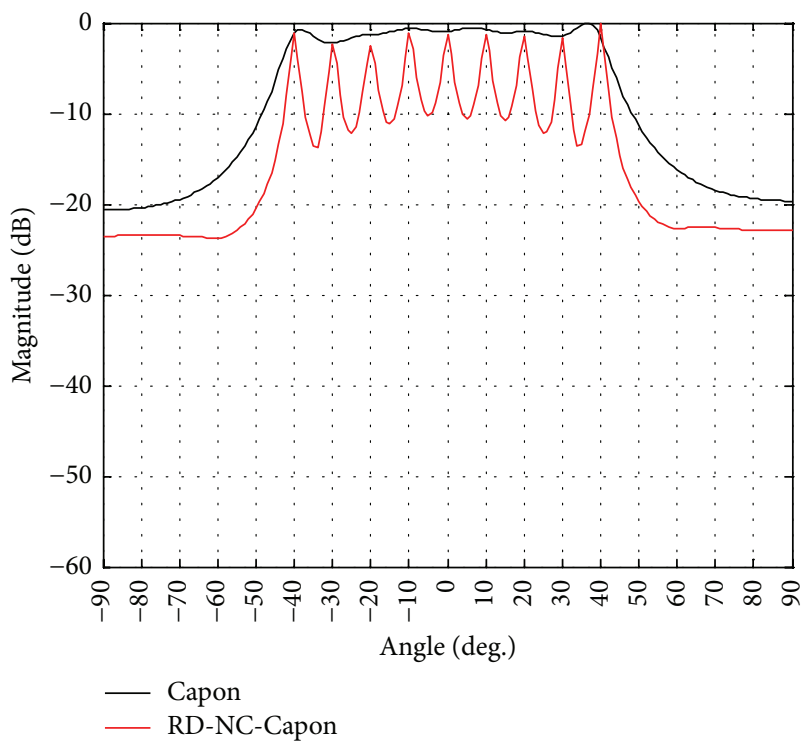

FIGURE 5: The spectrum search results of RD-NC-Capon algorithm $(\mathrm{SNR}=20 \mathrm{~dB}, M=8, L=200$, and $K=9)$.

the invariance shift property. Therefore, ESPRIT and NCESPRIT are not considered in Figure 6. Figure 7 shows the DOA estimation performance comparison among the ESPRIT algorithm, the NC-ESPRIT algorithm, the Capon algorithm, the 2D-NC-Capon algorithm, and the proposed algorithm for a uniform linear array with $\mathbf{d}=$ $[0,0.5 \lambda, 1.0 \lambda, 1.5 \lambda, 2.0 \lambda, 2.5 \lambda, 3.0 \lambda, 3.5 \lambda]$. From Figures 6 and 7 , we find that the $2 \mathrm{D}$-NC-Capon algorithm and the proposed algorithm have better angle estimation performance than the Capon algorithm. The reason is that the two NCCapon algorithms make full use of the noncircular property of the signals, and the array aperture is extended twice consequently. The proposed algorithm has very close DOA

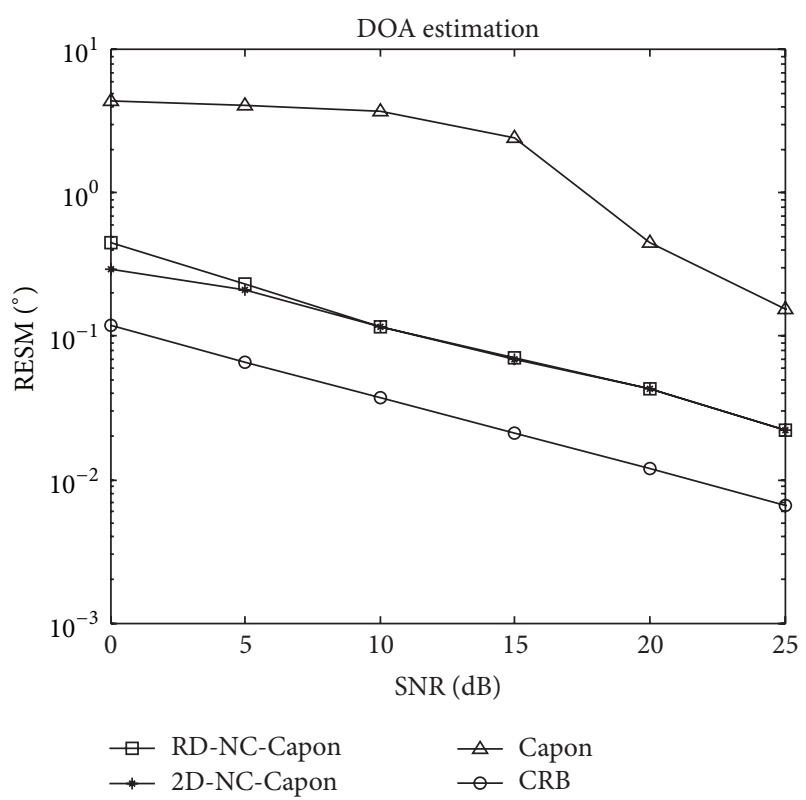

FIGURE 6: The DOA estimation performance comparison for nonuniform linear array $(M=8, L=200$, and $K=3)$.

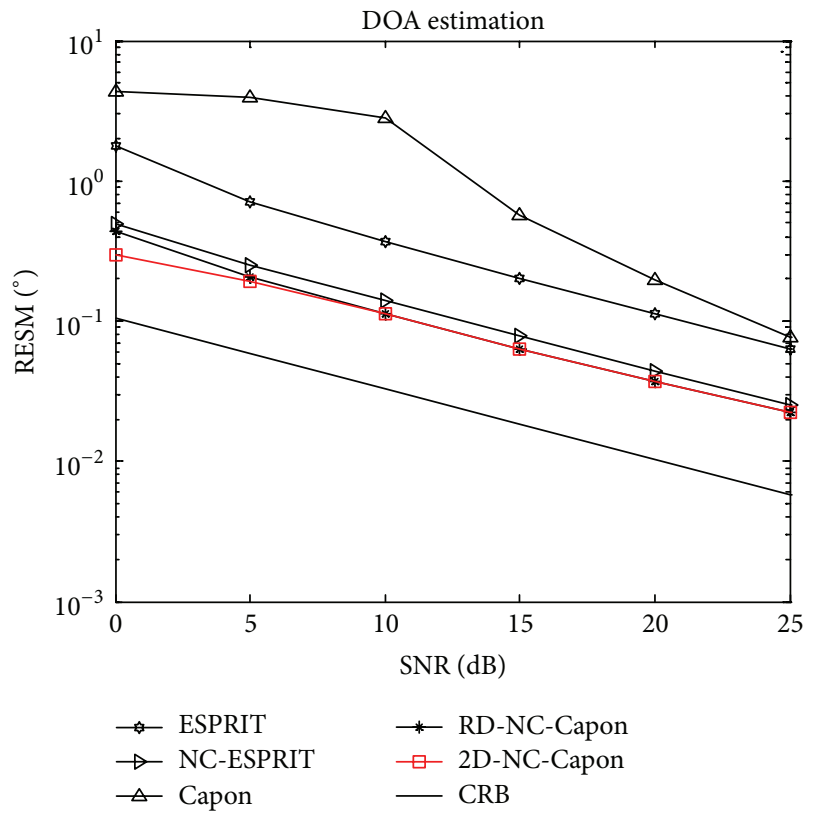

FIGURE 7: The DOA estimation performance comparison for uniform linear array $(M=8, L=200$, and $K=3)$.

estimation performance to the 2D-NC-Capon algorithm, which has a much higher complexity.

Figure 8 illustrates the DOA estimation performance of the proposed algorithm with different number of antennas $(M)$. It is clearly shown that the angle estimation performance of the proposed algorithm is gradually improved with the number of antennas increasing. Multiple antennas improve the DOA estimation performance because of antenna diversity gain. 


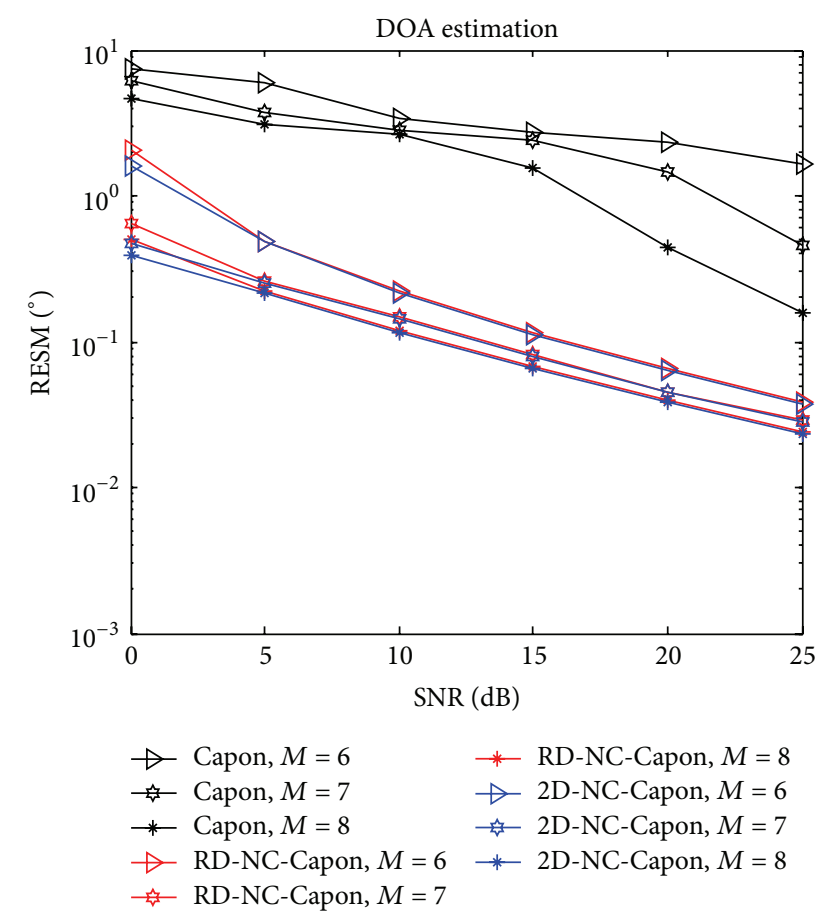

FIGURE 8: The DOA estimation performance with different numbers of antennas $(L=200$ and $K=3)$.

Figure 9 presents the DOA estimation performance of the proposed algorithm with different snapshots. In this simulation, $M=8, K=3$, and $\mathbf{d}=[0,0.45 \lambda, 0.9 \lambda, 1.3 \lambda$, $1.78 \lambda, 2.2 \lambda, 2.64 \lambda, 3.1 \lambda]$ are considered. From Figure 9, it is indicated that the DOA estimation performance becomes better with the snapshot increasing. The reason is that increasing the amount of sampled data makes the covariance matrix more accurate.

Figure 10 shows the spectrum search result of the proposed algorithm for UCA with SNR $=20 \mathrm{~dB}, M=8$, and $L=200$. The radii of the UCA are $0.7 \lambda$, and there are 3 noncircular signals impinging on UCA with angles being $\left(10^{\circ}, 25^{\circ}\right),\left(20^{\circ}, 15^{\circ}\right)$, and $\left(30^{\circ}, 35^{\circ}\right)$. From Figure 10, we find that the proposed algorithm works well.

\section{Conclusion}

In this paper, we have proposed the RD-NC-Capon algorithm for the DOA estimation of noncircular signals. Compared to the conventional Capon algorithm, the proposed RD-NCCapon algorithm has better estimation performance and can detect more sources by exploiting the noncircular property. Different from the 2D-NC-Capon algorithm, the proposed algorithm achieves DOA estimation with only a $1 \mathrm{D}$ search. It not only has much lower computational complexity but also has an angle estimation performance close to that of the 2D-NC-Capon algorithm, which has a much higher complexity than the proposed algorithm. Moreover, the proposed algorithm can be applied to arbitrary arrays and works well without estimating noncircular phases. Numerical

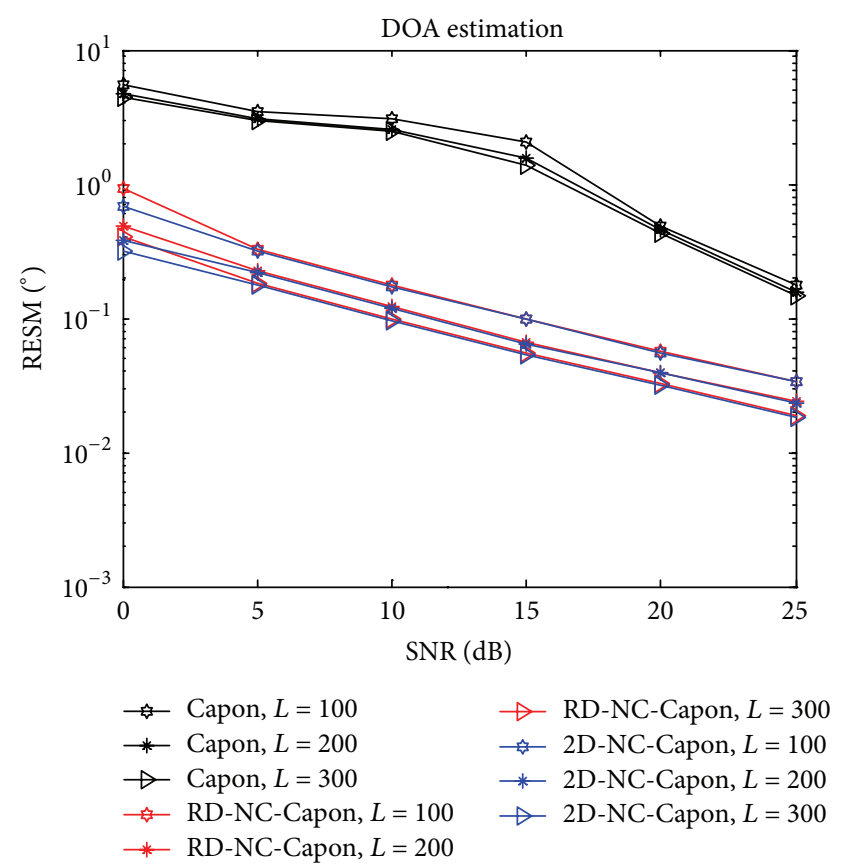

FIGURE 9: The DOA estimation performance with different number of snapshots $(M=8$ and $K=3)$.

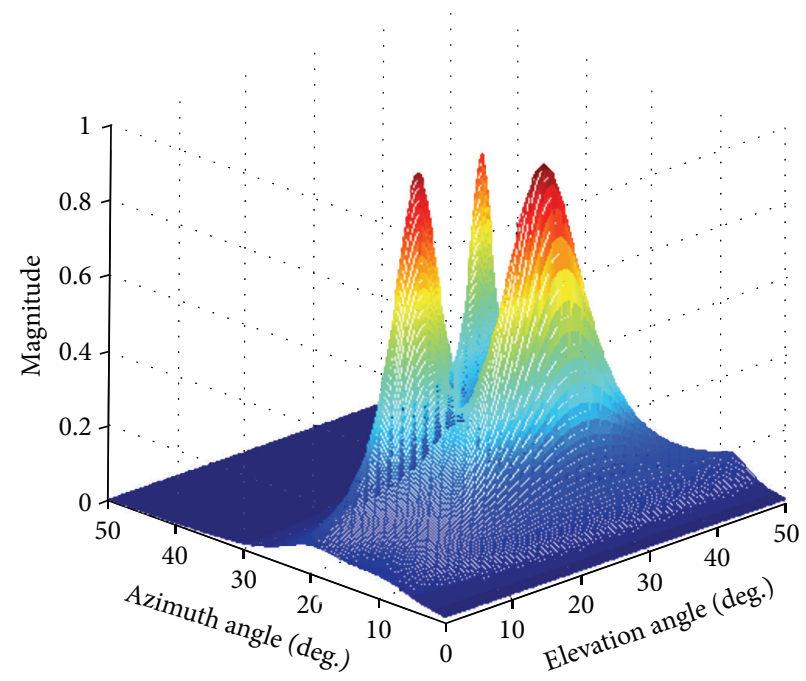

FIGURE 10: The spectrum search result of the proposed algorithm for the UCA.

simulation results verify the effectiveness and improvement of the proposed algorithm.

\section{Conflict of Interests}

The authors declare that there is no conflict of interests regarding the publication of this paper. 


\section{Acknowledgments}

This work is supported by China NSF Grants (61371169, $61301108,61471191,61471192$, and 61271327), the open research fund of National Mobile Communications Research Laboratory, Southeast University (no. 2015D03), Funding of Jiangsu Innovation Program for Graduate Education (KYLX15_0281), Funding for Outstanding Doctoral Dissertation in NUAA (BCXJ15-03), Qing Lan Project, and priority academic program development of Jiangsu high education institutions.

\section{References}

[1] H. Krim and M. Viberg, "Two decades of array signal processing research: the parametric approach," IEEE Signal Processing Magazine, vol. 13, no. 4, pp. 67-94, 1996.

[2] S. Applebaum, "Adaptive arrays," IEEE Transactions on Antennas and Propagation, vol. 24, no. 5, pp. 585-598, 1976.

[3] M. Zhou, X. Zhang, X. Qiu, and C. Wang, "Two-dimensional DOA estimation for uniform rectangular array using reduceddimension propagator method," International Journal of Antennas and Propagation, vol. 2015, Article ID 485351, 10 pages, 2015.

[4] H. Yu, X. Qiu, X. Zhang, C. Wang, and G. Yang, "Twodimensional direction of arrival (DOA) estimation for rectangular array via compressive sensing trilinear model," International Journal of Antennas and Propagation, vol. 2015, Article ID 297572, 10 pages, 2015.

[5] R. O. Schmidt, "Multiple emitter location and signal parameter estimation," IEEE Transactions on Antennas and Propagation, vol. 34, no. 3, pp. 276-280, 1986.

[6] G. Zheng, B. Chen, and M. Yang, "Unitary ESPRIT algorithm for bistatic MIMO radar," Electronics Letters, vol. 48, no. 3, pp. 179-181, 2012.

[7] M. L. Bencheikh and Y. Wang, "Joint DOD-DOA estimation using combined ESPRIT-MUSIC approach in MIMO radar," Electronics Letters, vol. 46, no. 15, pp. 1081-1083, 2010.

[8] M. D. Zoltowski, M. Haardt, and C. P. Mathews, "Closed-form 2D angle estimation with rectangular arrays in element space or beamspace via unitary ESPRIT," IEEE Transactions on Signal Processing, vol. 44, no. 2, pp. 316-328, 1996.

[9] S. Marcos, A. Marsal, and M. Benidir, "The propagator method for source bearing estimation," Signal Processing, vol. 42, no. 2, pp. 121-138, 1995.

[10] P. Stoica, P. Händel, and T. Söderström, "Study of Capon method for array signal processing," Circuits, Systems, and Signal Processing, vol. 14, no. 6, pp. 749-770, 1995.

[11] D. Model and M. Zibulevsky, "Signal reconstruction in sensor arrays using sparse representations," Signal Processing, vol. 86, no. 3, pp. 624-638, 2006.

[12] M. Carlin, P. Rocca, G. Oliveri, F. Viani, and A. Massa, "Directions-of-arrival estimation through Bayesian compressive sensing strategies," IEEE Transactions on Antennas and Propagation, vol. 61, no. 7, pp. 3828-3838, 2013.

[13] B. Picinbono, "On circularity," IEEE Transactions on Signal Processing, vol. 42, no. 12, pp. 3473-3482, 1994.

[14] H. Abeida and J.-P. Delmas, "MUSIC-like estimation of direction of arrival for noncircular sources," IEEE Transactions on Signal Processing, vol. 54, no. 7, pp. 2678-2690, 2006.
[15] P. Chargé, Y. D. Wang, and J. Saillard, "A non-circular sources direction finding method using polynomial rooting," Signal Processing, vol. 81, no. 8, pp. 1765-1770, 2001.

[16] A. Zoubir, P. Chargé, and Y. Wang, "Non circular sources localization with ESPRIT," in Proceedings of the 6th European Conference on Wireless Technology (ECWT '03), Munich, Germany, October 2003.

[17] J. Steinwandt, F. Roemer, and M. Haardt, "Performance analysis of ESPRIT-type algorithms for non-circular sources," in Proceedings of the 38th IEEE International Conference on Acoustics, Speech, and Signal Processing (ICASSP '13), pp. 3986-3990, Vancouver, Canada, May 2013.

[18] M. Haardt and F. Römer, "Enhancements of unitary ESPRIT for noncircular sources," in Proceedings of the IEEE International Conference on Acoustics, Speech, and Signal Processing (ICASSP '04), vol. 2, pp. 101-104, Montreal, Canada, May 2004.

[19] X. Sun and J. Zhou, "PM method for noncircular signals," Journal of Data Acquisition and Processing, vol. 28, no. 3, pp. 313318, 2013.

[20] X. Zhang, R. Cao, and M. Zhou, "Noncircular-PARAFAC for 2D-DOA estimation of noncircular signals in arbitrarily spaced acoustic vector-sensor array subjected to unknown locations," EURASIP Journal on Advances in Signal Processing, vol. 2013, no. 1, article 107, 2013.

[21] J. Liu, Z. T. Huang, and Y. Y. Zhou, "Azimuth and elevation estimation for noncircular signals," Electronics Letters, vol. 43, no. 20, pp. 1117-1119, 2007.

[22] J.-P. Delmas and H. Abeida, "Cramer-Rao bounds of DOA estimates for BPSK and QPSK modulated signals," IEEE Transactions on Signal Processing, vol. 54, no. 1, pp. 117-126, 2006.

[23] M. Wax and T. Kailath, "Detection of signals by information theoretic criteria," IEEE Transactions on Acoustics, Speech, and Signal Processing, vol. 33, no. 2, pp. 387-392, 1985.

[24] A. Di, "Multiple sources location-a matrix decomposition approach," IEEE Transactions on Acoustics, Speech, and Signal Processing, vol. 33, no. 5, pp. 1086-1091, 1985.

[25] H.-T. Wu, J.-F. Yang, and F. K. Chen, "Source number estimator using Gerschgorin disks," in Proceedings of the IEEE International Conference on Acoustics, Speech and Signal Processing (ICASSP '94), pp. IV/261-IV/264, Adelaide, Australia, April 1994.

[26] L. Huang, T. Long, E. Mao, and H. C. So, "MMSE-based MDL method for robust estimation of number of sources without eigendecomposition," IEEE Transactions on Signal Processing, vol. 57, no. 10, pp. 4135-4142, 2009.

[27] M. Zhong and Z. Fan, "Direction-of-arrival estimation for noncircular signals," in Proceedings of the International Conference on Computer Networks \& Communication Engineering, pp. 634$637,2013$.

[28] X. Zhang, F. Wang, H. Chen et al., Theory of Array Signal Processing and Its Applications (Version 2), Defense Industry Press, Beijing, China, 2013.

[29] X. Zhang and D. Xu, "Angle estimation in MIMO radar using reduced-dimension Capon," Electronics Letters, vol. 46, no. 12, pp. 860-861, 2010.

[30] P. Stoica and A. Nehorai, "Performance study of conditional and unconditional direction-of-arrival estimation," IEEE Transactions on Acoustics, Speech, and Signal Processing, vol. 38, no. 10, pp. 1783-1795, 1990.

[31] P. Stoica and R. Moses, Introduction to Spectral Analysis, Prentice-Hall, Upper Saddle River, NJ, USA, 1997. 

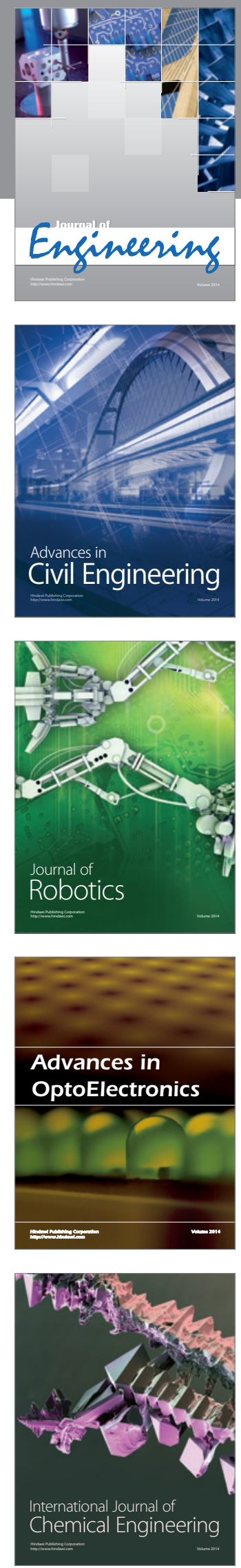

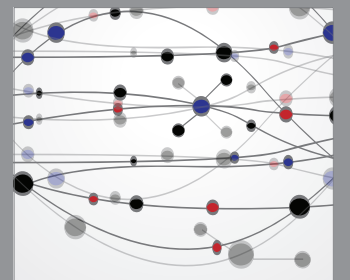

The Scientific World Journal
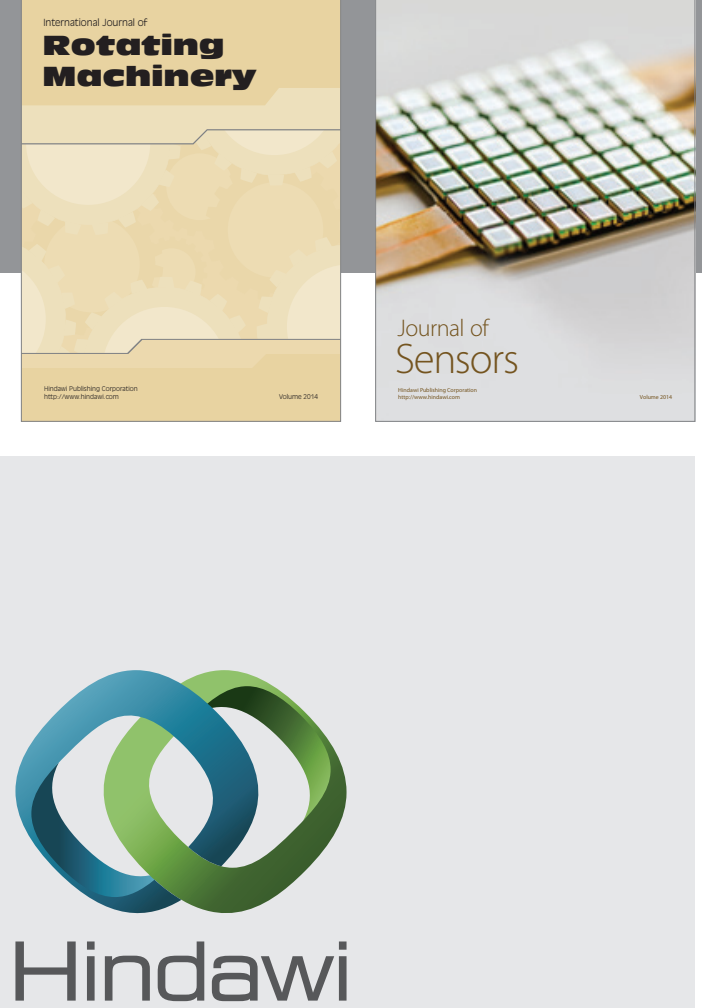

Submit your manuscripts at http://www.hindawi.com
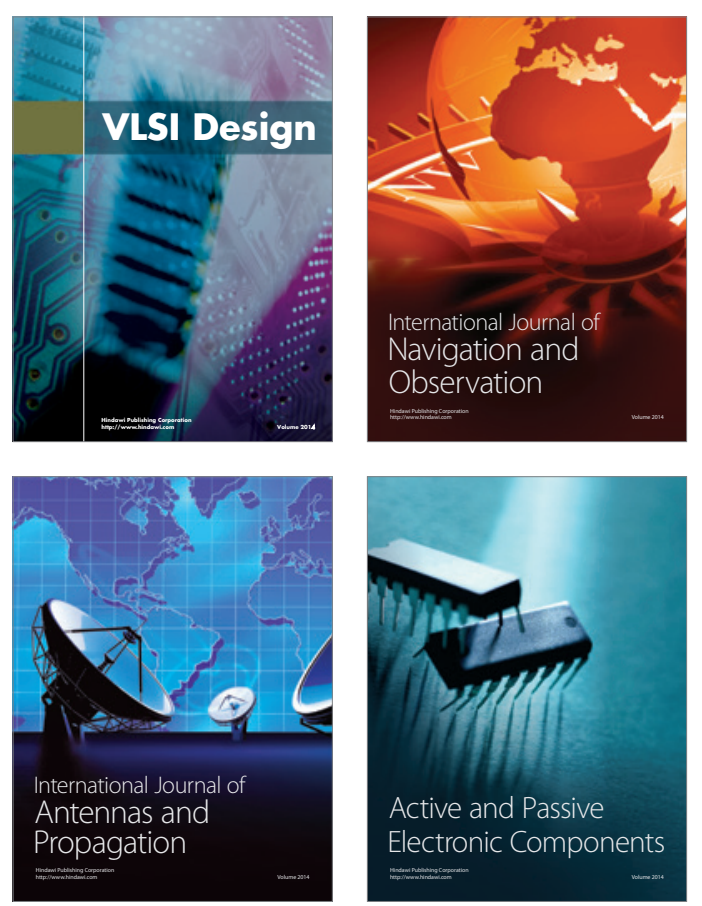
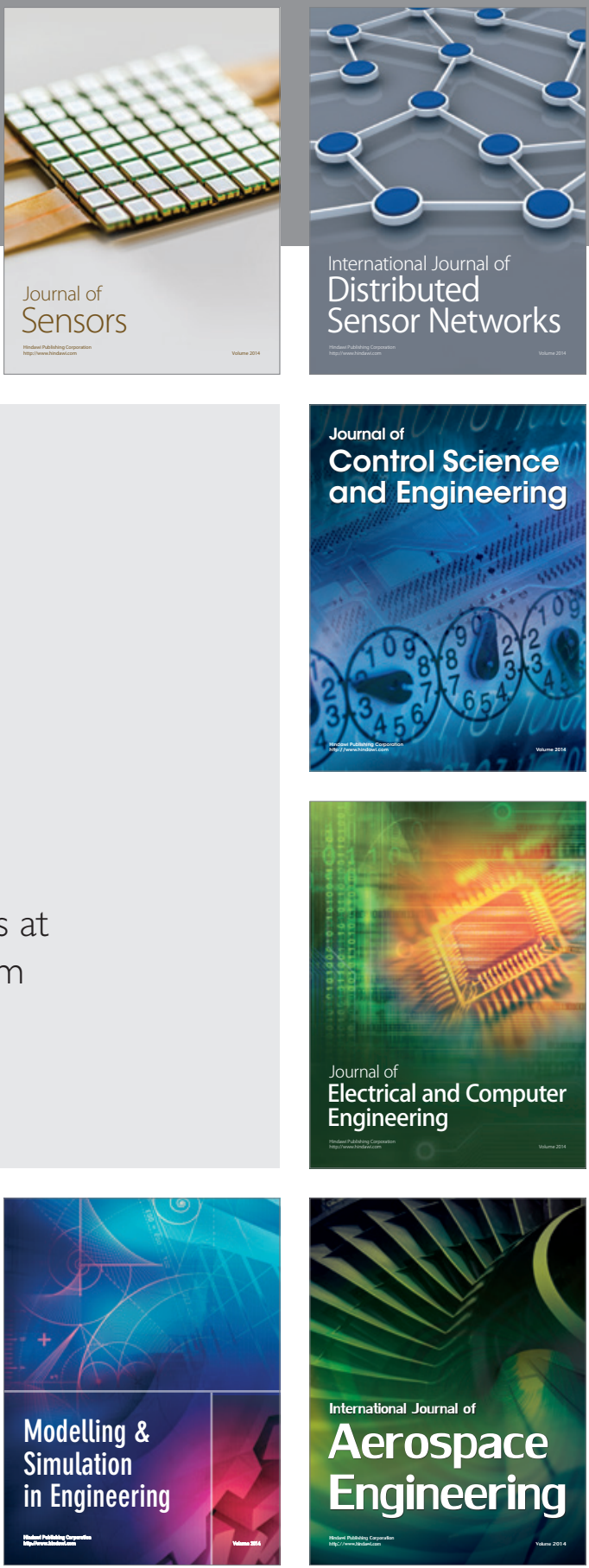

Journal of

Control Science

and Engineering
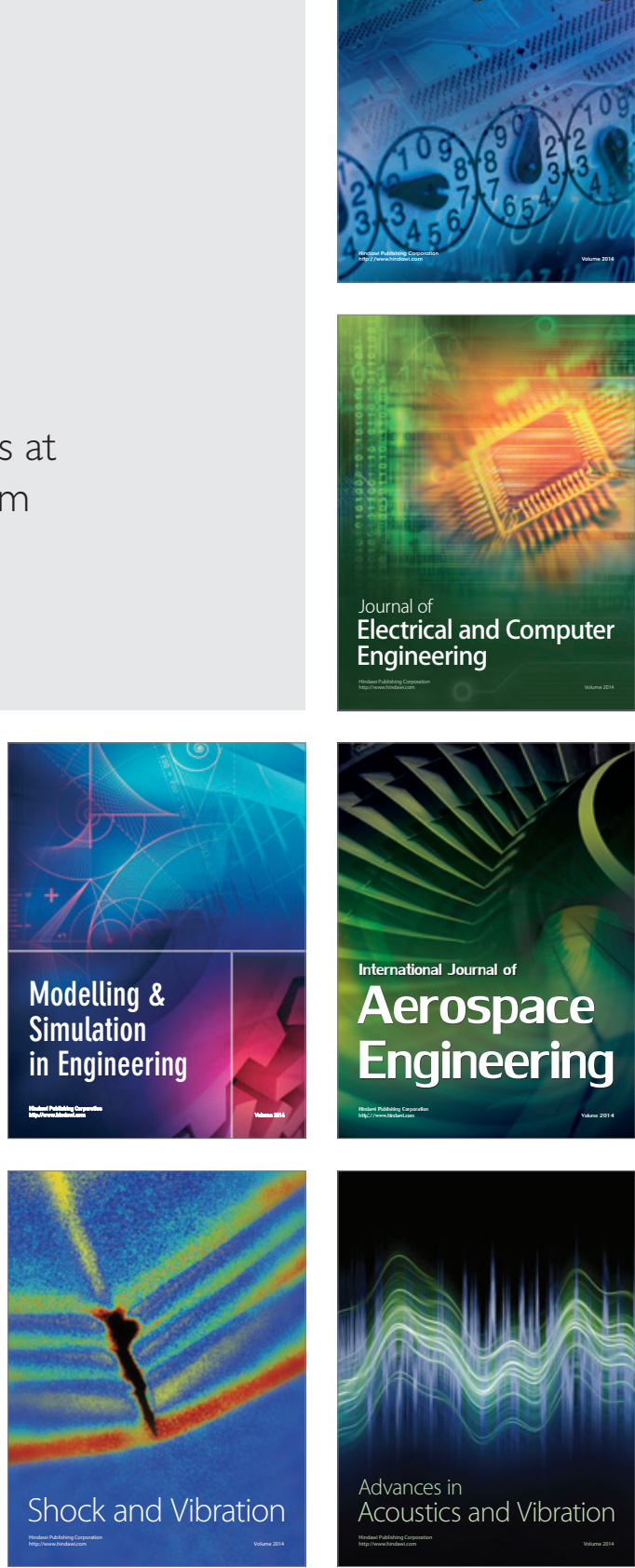